\title{
Microformatos: web 2.0 para el Dublin Core
}

Por Eva Méndez, Alejandro Bravo y Leandro Mariano López

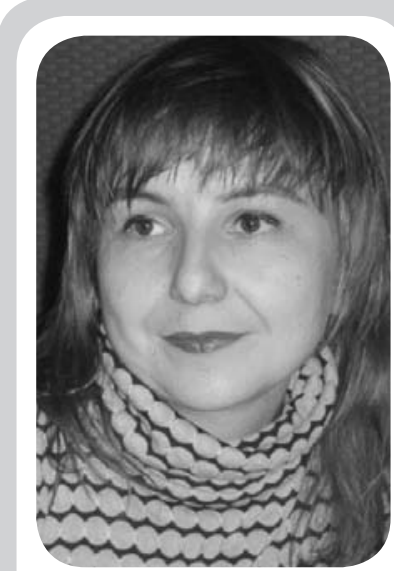

Eva Méndez es doctora en documentación y profesora del Departamento de Biblioteconomía y Documentación de la Universidad Carlos III de Madrid desde 1997. Participa activamente en distintos grupos de trabajo e investigación internacionales sobre la normalización de la Web y el acceso a la información digital y es miembro del Comité Asesor de la DCMI (Dublin Core Metadata Initiative). Durante el año académico 2005-06 fue investigadora en el Metadata Research Center de la Universidad de Carolina del Norte, Chapel Hill, EUA. Es autora de diversas publicaciones, principalmente sobre metadatos, estándares para la web semántica y gestión de contenidos digitales, temas en los que, entre otros, centra su investigación.

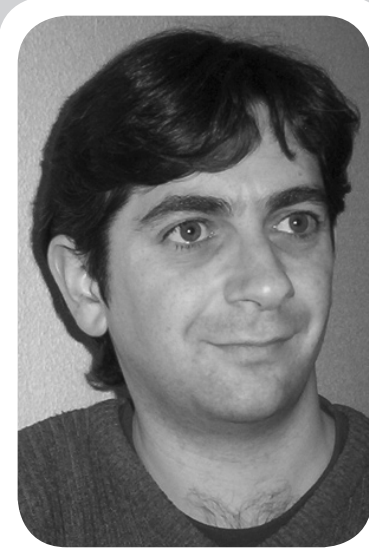

Alejandro Bravo es diplomado en estadística por la Universidad Complutense de Madrid, actualmente trabaja en Getronics como desarrollador web, fundamentalmente para clientes del sector financiero. Su interés por la web lespecialmente accesibilidad, web semántica, web móvil, estándares, etc.) le han llevado a mantener un sitio personal (Webposible) y sus blogs "Blog posible" y "Web es móvil".

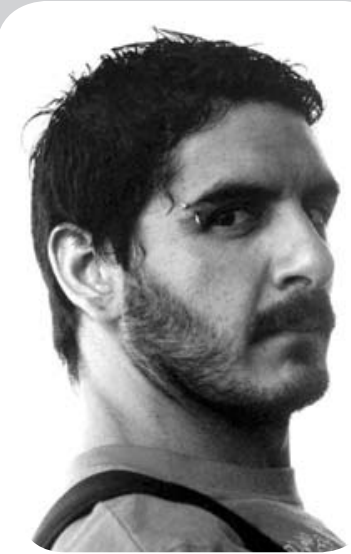

Leandro Mariano López, más conocido en la comunidad web como "inkel", trabaja como programador web en Buenos Aires (Argentina) y es además investigador freelance. En el año 2004 fue uno de los organizadores y ponentes del taller "La web semántica en América Latina". Participa activamente en grupos de trabajo y listas de correo sobre web semántica, metadatos, etc., siendo uno de los referentes en la comunidad hispanohablante interesada en estos temas.

Resumen: En este artículo se presenta el uso de microformatos Dublin Core (DC), una manera de utilizar el conjunto de elementos DC en una forma especial de marcado muy utilizada en la web 2.0 y denominada genéricamente "microformatos". Permiten la expresión de la semántica en el seno de una página xhtml de forma visible al usuario, estableciendo por tanto un alto nivel descriptivo para tipos de recursos concretos (tarjetas de visita, eventos, etc.). El caso concreto de los microformatos DC tiene un amplio potencial para la descripción de recursos en general, pero por su origen están especialmente indicados para describir recursos bibliográficos. Asimismo se presenta una forma sencilla de codificar DC en microformatos usando Dublin Core Metadata Gen, y otra aplicación que permite visualizar la información contenida dentro de estos micro-

formatos, a través de una extensión de Mozilla Firefox creada a tal efecto.

Palabras clave: Metadatos, Dublin Core, Web 2.0, Microformatos, Semántica embebida en la web, xhtml, RDF, Web semántica.

\section{Title: Microformats: web 2.0 for Dublin Core}

Abstract: This paper describes the use of Dublin Core (DC) microformats that allow the DC Metadata Element Set to be marked up in a style commonly applied in web 2.0. Microformats allow the semantic expression inside xhtml pages to be displayed in a manner visible to the user, thereby enabling a high level of description for specific types of resources (business cards, events, etc.). This particular use of DC microformats offers great potential for resource description in general, and is especially suitable for describing bibliographic resources. Also presented in this paper is a simple way for encoding DC in microformats using the Dublin Core Metadata Gen software, as well as an application that allows information included in the microformats to be displayed using the Mozilla Firefox extension, created for this purpose.

Keywords: Metadata, Dublin Core, Web 2.0, Microformats, Web embedded semantics, Xhtml, RDF, Semantic web.

Méndez, Eva; Bravo, Alejandro; Mariano López, Leandro. "Microformatos: web 2.0 para Dublin Core". En: El profesional de la información, 2007, marzo-abril, v. 16, n. 2, pp. 107-113.

DOI: 10.3145/epi.2007.mar.02

\section{Introducción}

EL SUEÑO DE LOS METADATOS INCLUIDOS en el código fuente de los documentos html se repite, pero ahora desde el punto de vista de los microformatos $^{1}$, una nueva forma de embeber semántica y metainformación en xhtml.
Cuando la web era sólo 1.0 (html era la única forma de representar y marcar la información, y Altavista era el buscador por excelencia) el único mecanismo aparentemente válido para incluir información semántica en los documentos eran las etiquetas $<$ meta $>$ o metaetiquetas. Esta metainformación debía introducirse en la cabecera 
de un documento html (entre las etiquetas $<$ head $>$ y $</$ head $>$ ), junto a otros elementos incluidos en el cuerpo del documento (entre $<$ body $>\mathrm{y}</$ body $>$ ) como title, address, link, del, ins, o los atributos title y cite que por su naturaleza eran especialmente adecuados para expresar información sobre la información. Desde un punto de vista práctico, los elementos <meta $>$ han sido, o han intentado ser, la forma más popular de añadir información semántica en los documentos xhtml. Pero su abuso y/o el mal uso (spamming) hizo fracasar el objetivo inicial de metadatos de propósito general aplicados a entornos de recuperación de información all-the-web -por búsqueda all-the-web entendemos aquella que se desarrolla en el seno de motores de búsqueda y servicios de recuperación tipo Google, que pretenden indizar de forma automática "toda la web"- o las propias metaetiquetas básicas $<$ meta keywords $=$ "" description $="$ "" $>$ debido a la utilización incoherente o abusiva de esa metainformación básica, que pronto sería ignorada por los motores de búsqueda.

Esta insuficiencia de expresión semántica llevaría a calificar a algunos autores a html como una norma envejecida e inflexible ${ }^{2}$. Sin embargo, con la popularización de xml y especialmente la estabilización de $r d f$ desde 1999 como una recomendación del $W 3 C^{3}$, se amplía la perspectiva de versatilidad de los metadatos y mejora notablemente su potencial y uso como uno de los elementos clave de la web semántica, en aras a la interoperabilidad y una recuperación de la información más precisa. Los microformatos y $r d f$ reviven el clásico debate entre los estándares de facto y de jure ${ }^{5} \mathrm{y}$ han llegado a denominarse "web semántica en minúsculas". Por ejemplo $r d f a$ es la nueva iniciativa (working draft) del $W 3 C^{25}$, que nace con vocación de estándar reconocido. Se trata de una sintaxis para expresar metadatos utilizando un conjunto de elementos y atributos que permiten embeber $r d f$ en xhtml. Mientras, los microformatos sirven básicamente para lo mismo, codificar metainformación en el seno de documentos xhtml, pero su uso extendido en la actualidad los ha convertido ya en un estándar de facto dentro de la denominada web 2.0.

http://www.w3.org/TR/xhtml2/

\section{"Los microformatos y rdf reviven el clásico debate entre los estándares de hecho y los oficiales, y han llegado a denominarse "web semántica en minúsculas"}

El objetivo principal de la web semántica es, de manera sencilla, conseguir un lugar más comprensible para las máquinas, pero también otro de sus objetivos, así como de los metadatos en particular, es hacer útiles los datos, directa o indirectamente, a las personas o usuarios de los mismos. Sin embargo, los metadatos referidos a páginas (metaetiquetas) no estaban dirigidos a las personas, sino más bien a los motores de indización y búsqueda. Así pues, cuando navegamos no los visualizamos directamente.

Recordemos que una página es, básicamente, un documento de texto plano fácilmente editable compuesto por etiquetas o tags, que puede contener atributos y sus valores determinados. En ella pueden aparecer más etiquetas o bien directamente el texto que se visualiza por el agente de usuario. De forma sencilla, la estructura básica de una página xhtml está compuesta por dos partes: la cabecera (<head $>$ y el cuerpo $(<$ body $>$ ). En la primera se pueden incluir metadatos, vínculos a ficheros javascript, vínculos relacionados, etc. $\mathrm{y}$, salvo en el caso de lo comprendido en la etiqueta $<$ title $>$ el contenido no se muestra generalmente en el navegador. El cuerpo está constituido por el contenido real de la página que el usuario ve en su pantalla.

Para poder acceder a la metainformación incluida en la cabecera tenemos, sin embargo, varias opciones:

- Visualizar el código fuente.

- Utilizar aplicaciones que la muestren, por ejemplo, configurando el navegador Opera, utilizando extensiones en Firefox, Mozilla o Seamonkey y que permitan la visualización de metadatos, como Meta Tags Sidebar ${ }^{6}$ o Dublin Core Viewer ${ }^{7}$, o bien a través de navegadores especializados con los que se pueden ver los metadatos embebidos en la cabecera al mismo tiempo que se accede al contenido (por ejemplo Metabrow$\left.\operatorname{ser}^{8}\right)$.

- Codificar los metadatos en el cuerpo (<body $>$ ) del documento de manera que sean mostrados ex profeso por el navegador (dirigidos al usuario).

Exactamente con esta última finalidad, mostrar información práctica a las personas, nacen los microformatos en el año 2003. Una forma de plantear una solución sencilla e innovadora a una cuestión sempiterna en la web: la nivelación entre la orientada al hombre y a la máquina. Con el objetivo de hacer útiles los datos, y mejorar la recuperación de información en la Red surgió el $D C$ en 1995 y, aunando ambos propósitos, nace la idea de codificar los elementos $D C$ en una sintaxis de microformatos $^{9,10}$.

\section{Microformatos: una forma de expresar metadatos en la web $\mathbf{2 . 0}$}

Tal y como se afirma directa y firmemente en la página del proyecto Microformats ${ }^{1}$, intentan ser útiles 
principalmente a las personas y en segundo lugar a las máquinas. Para ello aprovechan características de xhtml para añadir información semántica en una sección de su código.

Durante 2006, ha sido un tema recurrente en el blog de Stuart Weibel ${ }^{11,12}$, uno de los creadores del $D C$, el cual decía que "la flexibilidad que los microformatos permiten es una característica esencial de la hiper-innovación que caracteriza a la web 2.0", aunque también se preguntaba si encajarían en esta nueva web $^{11}$. La respuesta que damos en este artículo es sí, ya que permiten expresar la interacción que se demanda, conviviendo con la web tradicional. Hacen posible codificar las necesidades de la arquitectura participativa (folksonomías, redes sociales, etc.), con los estándares tradicionales (xhtml). Añadir expresiones de microformatos a páginas web permite a las máquinas procesar texto xhtml y también cargar información en bases de datos remotas, lo que a su vez permitirá a los buscadores encontrar información relativa, por ejemplo, a individuos o fechas relacionadas con una página web (una tarjeta de visita, un evento o un libro) descritos mediante microformatos. En definitiva, consultar usando semántica, que es, al fin y al cabo, la razón de ser implícita en los metadatos desde su origen, y explícita en la idea de web semántica.

\section{"La flexibilidad que los microformatos permiten es una característica esencial de la hiper-innovación que caracteriza a la web 2.0"}

Técnicamente, usar microformatos implica utilizar una codificación sencilla de los modelos o esquemas de metainformación para los atributos rel y rev, utilizados con los elementos $\langle a\rangle$ y $<$ link $>$ y los atributos id y class. El uso más habitual de estos dos últimos está relacionado con la presentación de documentos en la web. Pero también, según la especificación de html ${ }^{13}$, id tiene varios roles en html para propósitos generales de los agentes de usuario (por ejemplo para identificar campos cuando se extraen datos de páginas html en una base de datos, traduciendo documentos html a otros formatos, etc.). El atributo class, por su parte, asigna uno o más nombres de clase a un elemento, de tal forma que éste pertenece entonces a dichas clases. Un nombre de clase puede compartirse por varias instancias de elementos. El atributo class desempeña también varias funciones en html para propósitos generales que procesará el agente de usuario. Así pues, ambos atributos (class e id) tienen, una utilidad reconocida que transciende a su uso vinculado a los estilos de presentación CSS.
Un microformato no es más que un conjunto de valores "especiales" o finitos, usados con un propósito específico. La particularidad de estos valores es que normalmente forman parte de un conjunto determinado de elementos que, en ocasiones, está asociado a un estándar o esquema (schema) ampliamente adoptado, por ejemplo hCard y hCalendar o, como proponemos en este artículo, $D C$.

\subsection{Algunos microformatos: hCard, hCalendar, rel-tag}

Los microformatos ofrecen soluciones sencillas a problemas de representación y arquitectura de información concretos en la web, por ejemplo: cómo codificar la información personal de una tarjeta de visita, un evento o cómo ayudar a Technorati a "catalogar" mejor los artículos de los blogs.

\section{"Los microformatos ofrecen soluciones sencillas a problemas de representación y arquitectura de información concretos en la web"}

vCard e iCalendar $^{14}$ son dos estándares de descripción e intercambio de información, utilizados en diferentes aplicaciones y dispositivos como teléfonos móviles, PDAs o en aplicaciones para PC como Evolution o Palm Desktop. Se utiliza para la descripción de tarjetas de visita diferenciando nombre, dirección, teléfono, mail, página web, etc. e iCalendar para la descripción de eventos en el tiempo (citas, reuniones, congresos) con campos tan concretos como lugar, fecha de inicio y fin, etc. Mediante $h$ Card $^{15}$ e $h$ Calendar $^{16}$ se ha conseguido trasladar la versatilidad de $v$ Card e iCalendar al entorno de la web, convirtiéndose en microformatos abiertos adecuados para incluirse en xhtml, Atom, RSS, e incluso $\mathrm{xml}$.

Technorati es un buscador de artículos en blogs que basa la recuperación de información en etiquetas o tags. El proceso puede explicarse a través del siguiente ejemplo: un autor de blog publica un artículo sobre metadatos; el robot de Technorati lo encuentra y le asigna, de una forma automática etiquetas (tags) relacionadas con su contenido (por ejemplo metadatos, web semántica, etc.). Desde ese momento, cualquier persona interesada en la materia puede encontrar dicho artículo haciendo una sencilla búsqueda en Technorati. Todo este proceso, en principio automático, puede mejorar considerablemente si el propio autor del artículo o post, codifica las etiquetas que mejor lo describan, y así sea posible extraer sus contenidos para la indización temática. La idea del microformato rel-tag ${ }^{17}$, vincula- 
do a este buscador de blogs, persigue justamente eso, añadir rel="tag" a un hiperenlace, de tal forma que el destino de ese enlace es una indización de autor de la página que lo incluye. La forma de codificarlo en xhtml es muy sencilla, en nuestro ejemplo bastaría con añadir un vínculo en el artículo como este:

$<$ a ref.=http://technorati.com/tag/metadatos rel $=$ "tag" $>$ metadatos $</ a>$

De esta forma el microformato rel-tag, para clasificar el contenido de un artículo de blog, se une a los sistemas web 2.0 de etiquetado social o folksonomías, sistemas abiertos de indización/clasificación colaborativos y sin jerarquías específicas que reinan en sistemas como del.icio.us para la clasificación de favoritos, flickr.com para etiquetar fotos, o tagzania.com para clasificar lugares.

\section{Microformatos con DC}

\subsection{DC: un conjunto de elementos apto para mi- croformatos}

La Dublin Core Metadata Initiative (DCMI) es una organización dedicada a la promoción y difusión de estándares interoperables de metadatos y el desarrollo de vocabularios especializados para la descripción de recursos, de tal forma que permitan sistemas de recuperación más inteligentes. Una de sus principales actividades es el desarrollo y mantenimiento de un conjunto de elementos de metadatos "términos de metadatos" (Metadata-terms) que incluyen los 15 elementos reconocidos en la norma ISO 15836-2003 (contributor, coverage, creator, date, description, format, identifier, language, publisher, relation, rights, source, subject, title y type) y elementos o matizaciones de elementos (abstract, accessRights, accrualMethod, accrualPeriodicity, accrualPolicy, alternative, audience, available, etc. $)^{18}$.

Aprovechando por un lado la versatilidad, propósito general, nivel de normalización y difusión de los elementos de la DCMI y, por otro, la adecuación de los microformatos para expresar este tipo de metainformación, en el año 2005 se inició el proyecto de microformatos de $D C^{9}$. De esta forma unimos el conjunto de elementos y términos de metadatos del $D C$ a la lista de microformatos, ya que éstos permitirán potenciar las ventajas indiscutibles del $D C$ (simplicidad, flexibilidad y adecuación a cualquier dominio) para describir, a través de microformatos $D C$, cualquier recurso que se quiera citar en un documento web xhtml.

\subsection{Codificación de microformatos $D C$}

Aunque podrían utilizarse para citar online cualquier tipo de recurso en xhtml, pensemos en un libro; por ejemplo, la versión española de la obra de Tim

\section{"Microformatos y DC son una combinación perfecta para citar recursos en un documento web, de forma normalizada, simple, flexible y adecuada"}

Berners-Lee sobre cuya información bibliográfica básica es:

\section{Título: Tejiendo la red}

ISBN: 84-323-1040-9

\section{Autor: Tim Berners-Lee}

El código de representación de esta información en xthml usando microformatos $D C$ sería:

$$
\begin{aligned}
& <d l \text { class }=" \text { dublincore" }> \\
& <d t>\text { Título: }</ d t> \\
& <d d \text { class }=" t i t l e ">\text { Tejiendo la } \text { Red }</ d d> \\
& <d t>I S B N:</ d t> \\
& <d d \text { class }=" \text { identifier" }>84-323-1040-9</ d d> \\
& <d t>\text { Autor }:</ d t> \\
& <d d \text { class="creator" }>\text { Tim Berners }- \text { Lee }</ d d> \\
& </ d l>
\end{aligned}
$$

Según este ejemplo, el uso de microformatos Dublin Core, requiere:

a. Cualquier elemento contendedor de xhtml (en este caso una lista de definición $\langle d l\rangle$, probablemente el elemento más apropiado por su naturaleza), con la clase o identificador "dublincore" para identificar que el microformato que estamos usando es el $D C$.

b. Una cadena de texto que indique de forma inequívoca al usuario el concepto que se va a expresar a través del microformato (por ejemplo, el título). En el caso de codificar microformatos en xhtml en inglés, la mejor opción es utilizar directamente el nombre del elemento $D C$ (en el ejemplo, "title"). En el caso de que sea en otro idioma, como el español, la cadena de texto asociada al elemento $D C$ puede ser su traducción (por ejemplo "título").

c. Cualquier etiqueta de xhtml con una clase o identificador para codificar el conjunto de términos $D C^{18}$, cuyo valor de propiedad es uno de los elementos de la $D C M I$ (en el ejemplo, creator) y cuyo contenido es el valor de dicho elemento, en este caso Tim BernersLee.

También podrían considerarse a este nivel los llamados nanoformatos, donde el mismo elemento html sirve como contenedor de metadatos y como metainformación en sí mismo: 
$<$ span class="dublincore modified">2006-12$06</$ span $>$

\subsection{Microformatos, etiquetas $<$ meta $>$ y ficheros $r d f$}

Los microformatos son una forma más de expresar los metadatos dentro de un recurso electrónico. Si los comparamos con las etiquetas <meta $>$ y los ficheros $r d f$, presentan algunas ventajas, o al menos particularidades, de las que cabe destacar:

- La información se muestra directamente a los usuarios.

- Son sencillos de codificar para cualquier creador/ desarrollador de páginas web, en tanto que no implican el conocimiento de un nuevo lenguaje, sino la utilización oportuna de los estándares existentes (elementos y atributos de html que ya conoce, y un conjunto de valores propios de cada tipo de microformato $(D C$, por ejemplo).

- Los datos visibles son probablemente más útiles para las personas, y más precisos a largo plazo, ya que los posibles errores pueden corregirse más fácilmente que cuando se trata de metadatos ocultos.

Sin embargo, existen algunas limitaciones, e incluso quizás ciertos riesgos, para la generalización de su uso:

- Son menos conocidos que las etiquetas $\langle$ meta $>$, ya que pertenecen al dominio emergente, aunque tan de moda, de la web 2.0.

- Aunque existe un lenguaje para crear perfiles para trabajar con microformatos - Xmdp (Xhtml meta data profiles $\left.{ }^{19}\right)$ - no están tan formalmente definidos como ocurre con los profile asociados al trabajo con etiquetas <meta $>$ o los propios esquemas incluidos en espacios de nombre (namespaces) de los ficheros $r d f$.

- Si comparamos los microformatos con los ficheros $r d f$, tienen una menor capacidad de expresión semántica que éstos. Por ejemplo, no existe la posibilidad de definir formalmente relaciones complejas que puedan servir para que los agentes de software realicen inferencias semánticas o deducciones sobre su contenido.

\subsection{Cómo crear microformatos DC: Dublin Core Metadata Gen}

Si bien existen diversas herramientas para la extracción y/o generación de metadatos $D C^{20}$ que contemplan distintas sintaxis de codificación y/o distintos formatos de salida ninguna de ellas, hasta ahora, permite como opción codificar los elementos como microformatos.

Dublin Core Metadata Gen ${ }^{20}$ es una aplicación desarrollada en xhtml y javascript que contempla esta posibilidad, permitiendo generar metadatos $D C$ de tres formas distintas: contenido de un fichero $r d f$, etiquetas $<$ meta> de xhtml y también microformatos $D C$.

Se trata de una aplicación tipo plantilla en la que el creador de los metadatos introduce los valores necesarios para completar los elementos del $D C$, a través de un formulario (título, autor, descripción, etc.), y al generarlos obtiene, en forma de tres áreas de texto, $D C$ en $r d f$, en metaetiquetas xhtml y en microformatos.

\subsection{Visualización de información contenida en microformatos $D C$ mediante el visor de microfor-} matos $D C$

$\mathrm{Si}$ entendemos los microformatos como una forma de codificación, primero para las personas y después para las máquinas, surge otra importante cuestión ¿Cómo visualizan las personas los microformatos $D C$ ? Con esa intención surge la extensión "visor de microformatos DC" para los navegadores Firefox y Flock ${ }^{22}$. Su funcionamiento es sencillo y muy parecido a Dublin Core Viewer Extensión ${ }^{7}$ y, al igual que éste, también es de código abierto. Una vez instalada, la extensión se integra en el agente de usuario y presenta un indicador en

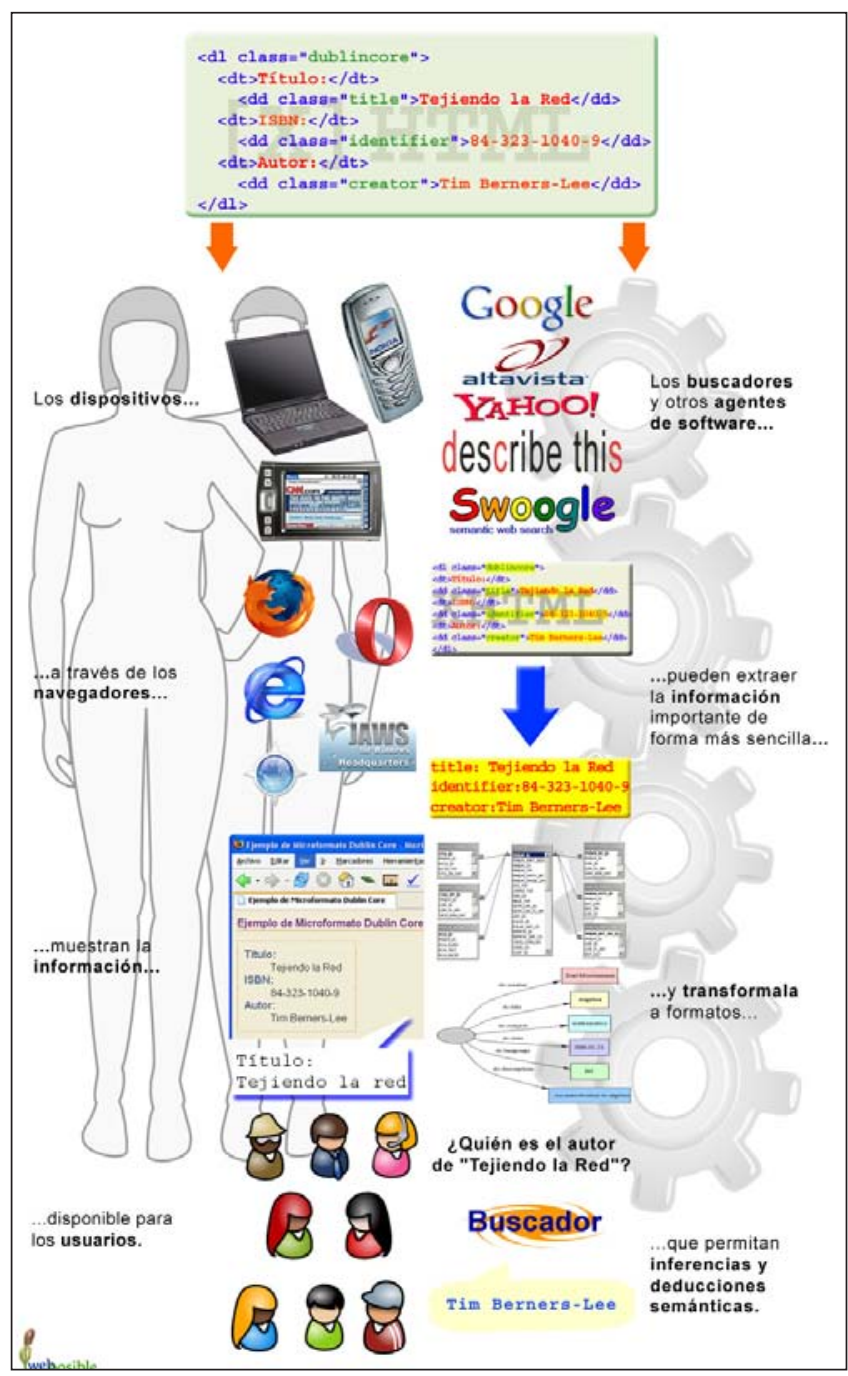

Figura 1. Beneficios de los microformatos para las personas y para las máquinas 
la barra de estado donde se informa si la página actual que estamos navegando posee o no microformatos $D C$. Cuando se encuentran (figura 2), se puede presionar un botón, desplegando una nueva ventana donde se listan junto a sus valores para cada elemento $D C$, como si fuese una pequeña ficha bibliográfica.

Es una extensión sencilla en su funcionalidad, que al mismo tiempo permite darnos una idea de la facilidad de obtener metainformación a partir del contenido mismo del documento. Si los agentes de usuario y de software son capaces de reconocerla, ya que se encuentra codificada en microformatos, y de visualizarla, será más fácil que se recupere y se utilice para distintos propósitos: indización por parte de buscadores, recodificación en otros formatos ( $r d f$, bases de datos, etc.), establecer relaciones complejas o inferencias semánticas, entre otras cosas.

\section{Conclusiones y trabajo futuro}

El tema de cómo, cuándo y quién crea metadatos $D C$ no es nuevo, como tampoco lo es el argumento de la simplicidad del $D C$ para que el propio autor del recurso web cree datos y metadatos ${ }^{23}$. La pobreza semántica en la codificación de metadatos a través de etiquetas <meta>, llevó al W3C a crear $r d f$ bajo la inspiración de la idea de web semántica. Sin embargo la web 2.0, más semántica también, necesita formas flexibles para codificar el significado y la interacción que la caracterizan. En este sentido se han analizado aquí los microfor- matos como una nueva forma de pensar, no sólo sobre los datos, sino también sobre los metadatos.

Los microformatos son una realidad, de gran aceptación en algunos casos (rel= "tag" en Technorati, como hemos comentado aquí, o rel="no-follow" que tiene en cuenta Google ${ }^{24}$ ), ya que han resuelto con facilidad problemas concretos. Al ser perfectamente compatible con recomendaciones del $W 3 C$ (html, xhtml, css), su uso no impide que el código sea válido y pueden ser perfectamente integrados en cualquier página web.

Los microformatos $D C$ son además, una forma de resolver con facilidad la descripción y citación online de recursos, poniendo de manifiesto nuevamente la sencillez y versatilidad de $D C$ como un modelo de metadatos de propósito general. Asimismo son una solución interesante tanto desde el punto de vista del desarrollador, como del de los agentes de usuario o de software.

Futuros trabajos en el tema de microformatos $D C$ podrían incluir el desarrollo de las aplicaciones mencionadas en este artículo para propósitos particulares, por ejemplo adaptando el uso de DC Metadata $G^{21}{ }^{21}$ a la gestión bibliográfica de citaciones; o la adaptación del visualizador de microformatos $D C^{22}$ a agentes de software de minería de microformatos; e incluso, el propio análisis del uso de microformatos $D C$ y su potencialidad para el establecimiento de análisis bibliométrico de la web en entornos de archivos abiertos o de la valiosa información del mundo del blogging, donde las citas a otros recursos (páginas, posts, etc.) es tan habitual.

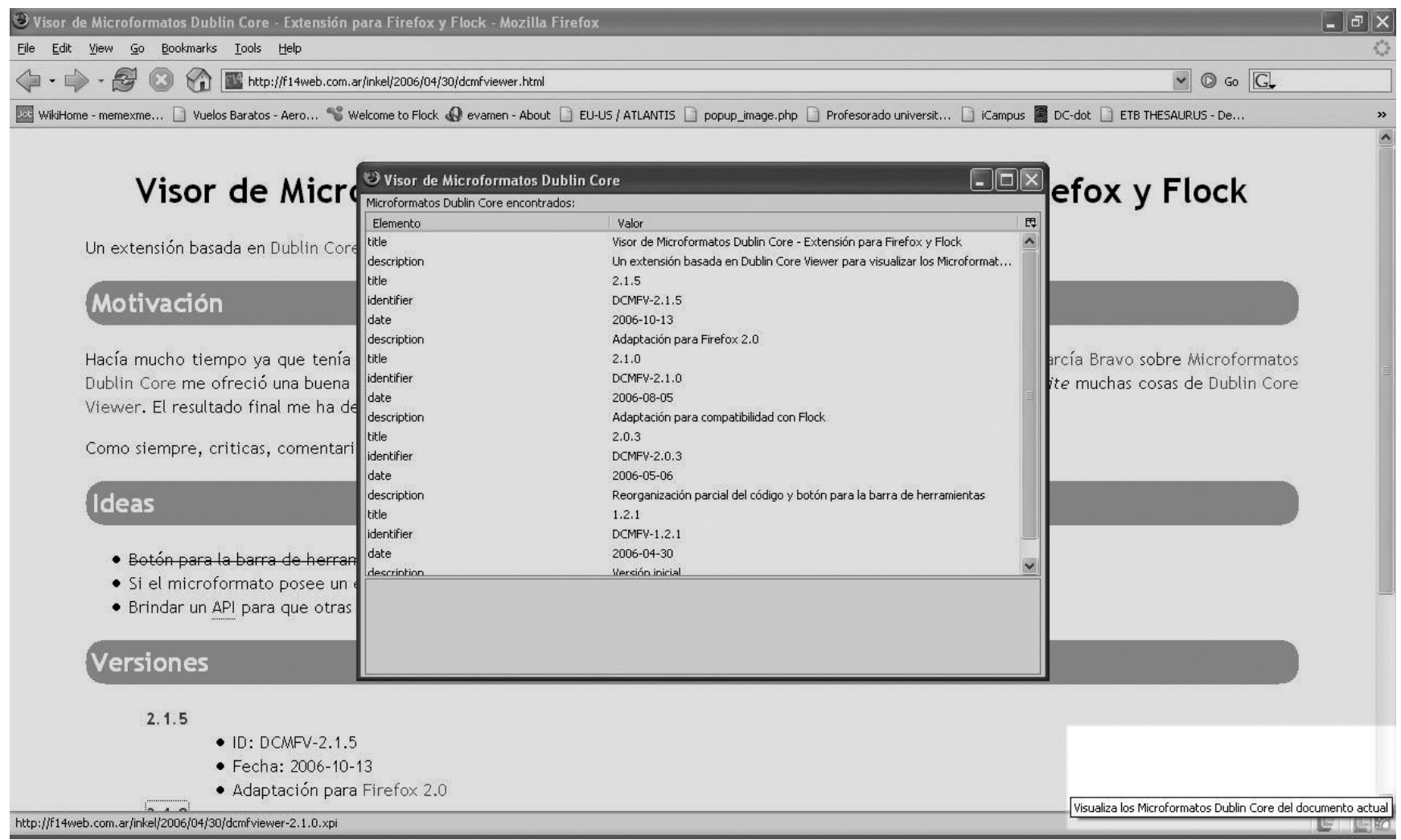

Figura 2. Funcionamiento del visor de microformatos DC (Firefox extension) 


\section{"Grddl será un estándar que dará mucho que hablar en el futuro, tratando de aunar lenguajes y dialectos de la web 2.0, 3.0., etc."}

El uso de los microformatos en general se debe a su simplicidad, y a su aceptación y adopción en el ámbito de sistemas web 2.0. Sin embargo, es preciso también prestar atención a las nuevas iniciativas para la inclusión y manejo de metadatos en contextos web 2.0, tales como $r d f a^{25,5}$. Además el $W 3 C$ trata de aunar los sistemas de definición semántica más formales con otros, como los microformatos que, de una forma menos estricta y ágil, revisten de semántica adicional a documentos xhtml válidos. En este sentido Grddl (Gleaning resource descriptions from dialects of languages $)^{26}$ es un mecanismo para obtener la descripción de los recursos a partir de lenguajes (por ejemplo xht$\mathrm{ml}$ ) y dialectos (microformatos o $r d f a$ ). Se trata de una forma de extraer $r d f$ fuera de documentos xml o xhtml a través de algoritmos de transformación representados en el lenguaje de estilos de transformación xml (XSLT). Sin duda alguna $G r d d l$, será un estándar que dará mucho que hablar en el futuro, en la web 2.0 y 3.0.

\section{Referencias}

1. Microformats.

http://microformats.org

2. Kiely, D. "Xhtml: a bridge to the future". En: Information week online Consultado en: 09-10-06.

http://www.informationweek.com/785/language.htm

3. World Wide Web Consortium. Resource description framework ( $r d f)$ model and syntax specification. W3C recommendation 22 February 1999. Lassila, Ora; Swick, Ralph R. (eds.), W3C. Consultado en: 13-11-06. http://www.w3.org/TR/1999/REC-rdf-syntax-19990222

4. Noriega, Manuel G. Microformatos: la web semántica en minúsculas. Consultado en 20-11-06.

http://www.alzado.org/articulo.php?id_art $=416$

5. Prodromou, Evan. Rdfa vs microformats. Consultado en: 20-11-06. http://evan.prodromou.name/RDFa_vs_microformats

6. Meta Tags Sidebar (Version 2.3.5, released on Jan 4, 2006).

https://addons.mozilla.org/firefox/1668

7. Lauke, Patrick H. Firefox Dublin Core viewer extension. http://www.splintered.co.uk/experiments/73

8. Metabrowser (Metabrowser Systems, Australia).

http://metabrowser.spirit.net.au

9. Bravo, Alejandro G. Microformatos Dublin Core. En: Webposible. http://www.webposible.org/microformatos-dublincore

10. Gipp, Siegfried. Combining the ideas of microformats and Dublin Core. http://www.rorkvell.de/tech/dc

11. Weibel, Stuart. Ockham's bathroom scale, Lego blocks, and microformats. Consultado en: 13-04-06.
12. Weibel, Stuart. Microformats again. Consultado en: 10-11-06. http://weibel-lines.typepad.com/weibelines/2006/11/microformats_ag.html

13. Raggett, D.; Le Hors, A.; Jacobs, I. (eds.). World Wide Web Consortium. Html 4.01 specification: W3C Recommendation 24 December 1999, W3C. Consultado en: 05-11-06.

http://www.w3.org/TR/html401

14. vCard and iCalendar (Internet Mail Consortium).

http://www.imc.org/pdi/

vCard, RFC 2426

http://www.ietf.org/rfc/rfc2426.txt

iCalendar también es un estándar del IETF, Internet calendaring and scheduling core object specification (RFC 2445).

http://www.ietf.org/rfc/rfc2445.txt

15. Microformato hCard.

http://microformats.org/wiki/hcard

16. Microformato hCalendar.

http://microformats.org/wiki/hcalendar

17. Microformato rel-tag.

http://microformats.org/wiki/rel-tag

18. Dublin Core Metadata Terms.

http://dublincore.org/documents/dcmi-terms

19. XMDP: Xhtml meta data profiles.

http://www.gmpg.org/xmdp

20. DC-Tools

http://dublincore.org/tools

21. Bravo, Alejandro G. Dublin Core Metadata Gen. En: Webposible. http://www.webposible.com/utilidades/dublincore-metadata-gen

22. López, Leandro M. Visor de microformatos Dublin Core: extensión para Firefox y Flock.

http://f14web.com.ar/inkel/2006/04/30/dcmfviewer.html

Última versión para Firefox (DCMFV-2.1.5; 13-10-06).

http://f14web.com.ar/inkel/2006/04/30/dcmfviewer-2.1.5.xpi

Última versión para Flock (DCMFV-2.1.0; 05-08-06).

http://f14web.com.ar/inkel/2006/04/30/dcmfviewer-2.1.0.xpi

23. Méndez Rodríguez, Eva. "El problema de la asignación de metadatos". En: Metadatos y recuperación de información, estándares, problemas y aplicabilidad en bibliotecas digitales. Gijón: Trea, 2002, (capítulo 2). Isbn 84-9704-055-4.

24. Preventing comment spam

http://googleblog.blogspot.com/2005/01/preventing-comment-spam.html

25. Adida, Ben; Birbeck, Mark (eds.). World Wide Web Consortium. Rdfa Primer 1.0-Embedding rdf in xhtml: W3C working draft 16 May 2006. Consultado en: 01-12-06.

http://www.w3.org/TR/xhtml-rdfa-primer/

26. Connolly, Dan (ed.). World Wide Web Consortium. Gleaning Resource Descriptions from Dialects of Languages (Grddl): W3C Working Draft 24 October 2006. Consultado en: 01-12-06.

http://www.w3.org/TR/grddl/

Eva Méndez, Departamento de Biblioteconomía y Documentación de la Universidad Carlos III de Madrid. emendez@bib.uc3m.es

Alejandro Bravo, Webposible. alejandrogbravo@yahoo.es

Leandro Mariano López.

inkel-rdf@fl4web.com.ar 